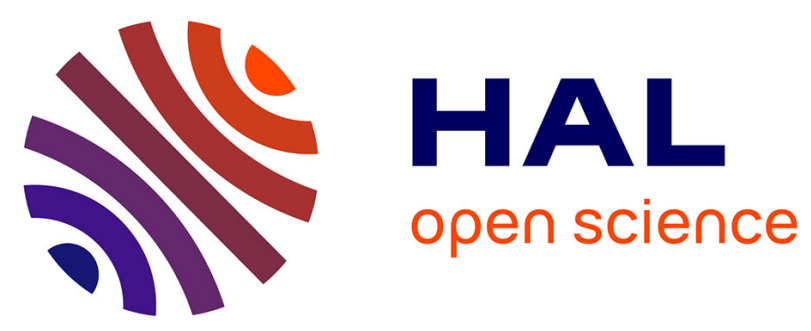

\title{
Performances du ratio lactate/glucose synovial pour le diagnostic d'arthrite septique
}

O. Berthoud, G. Coiffier, J.-D. Albert, A. Gougeon-Jolivet, C. Goussault, C. Bendavid, P. Guggenbuhl

\section{> To cite this version:}

O. Berthoud, G. Coiffier, J.-D. Albert, A. Gougeon-Jolivet, C. Goussault, et al.. Performances du ratio lactate/glucose synovial pour le diagnostic d'arthrite septique. Revue du Rhumatisme, 2021, 88 (2), pp.138-145. 10.1016/j.rhum.2021.01.004 . hal-03196164

\section{HAL Id: hal-03196164 https://hal.science/hal-03196164}

Submitted on 13 Apr 2021

HAL is a multi-disciplinary open access archive for the deposit and dissemination of scientific research documents, whether they are published or not. The documents may come from teaching and research institutions in France or abroad, or from public or private research centers.
L'archive ouverte pluridisciplinaire HAL, est destinée au dépôt et à la diffusion de documents scientifiques de niveau recherche, publiés ou non, émanant des établissements d'enseignement et de recherche français ou étrangers, des laboratoires publics ou privés. 
Performances d'un nouveau test rapide, le ratio lactate/glucose synovial pour le diagnostic d'arthrite septique.

Olivia Berthoud a , Guillaume.Coiffier a,b, Jean-David Albert a,b, Anne Gougeon-Jolivet ${ }^{\mathrm{b}, \mathrm{c}}$, Claire Goussault a, Claude Bendavid d,e, Pascal Guggenbhula,b

aService de Rhumatologie, Centre Hospitalier Universitaire de Rennes - Pontchaillou, 2 Rue Henri Le Guilloux, 35000, Rennes, France.

bUMR INSERM U 1241, Université de Rennes, Rennes, France

cLaboratoire de Bactériologie, Centre Hospitalier Universitaire de Rennes - Pontchaillou, 2 Rue Henri Le Guilloux, 35000, Rennes, France.

aService de Biochimie, Centre Hospitalier Universitaire de Rennes - Pontchaillou, 2 Rue Henri Le Guilloux, 35000, Rennes, France.

eINSERM Unité Mixte de Recherche 991, F-35043 Rennes, France

Auteur correspondant : Olivia Berthoud

olivia.berthoud@chu-rennes.fr

02.99 .26 .71 .40 


\begin{abstract}
:
Objectif : Évaluer les performances diagnostiques du dosage du lactate, du glucose et du ratio lactate/glucose synovial pour le diagnostic d'arthrite septique.

Méthodes : Dans cette étude transversale monocentrique, les liquides synoviaux de patients souffrant d'un épanchement articulaire aigu ( $<30$ jours) sur articulations natives ont été recueillis de manière prospective. L'arthrite septique a été définie selon les critères de Newman. Pour évaluer les performances diagnostiques, ont été calculés la courbe ROC (Receiver Operating Characteristic) avec l'aire sous la courbe (AUC), la sensibilité $(\mathrm{Se})$, la spécificité $(\mathrm{Sp})$ et le rapport de vraisemblance positif $(\mathrm{RV}+)$ avec leur intervalle de confiance à $95 \%$. La culture bactériologique des liquides synoviaux avec coloration de Gram, la recherche de microcristaux, la numération leucocytaire (GBsyn) et le dosage du glucose et du lactate ont été réalisés.
\end{abstract}

Résultats : Au total, 233 liquides synoviaux ont été inclus comprenant 25 arthrites septiques et 208 arthrites non septiques (104 arthrites microcristallines, 15 PR, 8 SpA, 6 arthrites réactionnelles et 75 arthrites aiguës d'origine indéterminée). Le ratio lactate/glucose synovial était plus haut que le dosage séparé du lactate et du glucose synovial (AUC : 0.859 [0.772-0.945]). La valeur seuil idéale pour le ratio lactate/glucose synovial permettant de différencier l'arthrite septique de l'arthrite non septique était de 5 Se $52 \%$ [0.34-0.7], Sp 98.1 \% [0.95-0.99], RV+ $27.0[9.50-76.00])$.

Conclusion : Les performances diagnostiques du ratio lactate/glucose synovial permettent de discriminer de manière efficace et très rapide les arthrites septiques des autres formes d'arthrite.

\title{
Mots clés :
}

Arthrite septique

Glucose

Liquide synovial

Ratio lactate/glucose

Lactate

Arthrite aiguë 


\section{Introduction}

La survenue d'une arthrite septique est une urgence médicale. En effet seule l'instauration sans délai d'une antibiothérapie efficace permet de réduire le risque élevé de sepsis compromettant le pronostic vital et de destruction articulaire grévant le pronostic fonctionnel (1-6). La différenciation de l'arthrite septique d'une autre arthrite inflammatoire aiguë peut être complexe en raison de leurs manifestations cliniques et biologiques aspécifiques (7-9). Si elle reste une étape essentielle pour l'identification du pathogène, la mise en culture du liquide synovial est une démarche longue qui retarde l'instauration d'une antibiothérapie ciblée (10). La coloration de Gram est une méthode rapide mais qui présente une sensibilité limitée pour détecter l'infection bactérienne, à savoir d'environ 45 à $50 \%(4,11-13)$. La culture du liquide synovial peut être négative dans $20 \%$ des cas et nécessite un délai pouvant aller jusqu'à 72 heures.

L'identification d'un biomarqueur synovial disponible en routine clinique semble utile pour discriminer une arthrite septique plus rapidement et améliorer sa prise en charge. De nombreux marqueurs comme le nombre de globules blancs, le pourcentage de polynucléaires (PNN) synoviaux, l'interleukine-6 et la procalcitonine synoviale ont été analysés sans parvenir à un consensus concernant leur intérêt diagnostique (13-20)

Cependant, le métabolisme bactérien a fait l'objet d'études approfondies. Ainsi, il a été montré que la présence d'une bactérie génère la consommation de glucose et par là même la production d'acide lactique. Le lactate et le glucose sont des biomarqueurs diagnostiques connus depuis de nombreuses années. Peu utilisés entre 1970 et 2000 , leur étude soulève désormais un intérêt scientifique (21). Ces biomarqueurs sont utilisés tout particulièrement dans le diagnostic de la méningite bactérienne et affichent de bonnes performances diagnostiques (22). Dans le diagnostic d'arthrite septique, leur utilisation pourrait également permettre de poser le diagnostic plus précocement que la mise en culture standard (23-25). Pour surmonter les contraintes de temps, des techniques de dépistage rapide, à savoir l'utilisation de bandelettes urinaires et du glucomètre directement dans le liquide synovial, ont été évaluées (26-28).

Cette étude avait pour principal objectif d'évaluer l'intérêt clinique et les performances diagnostiques du dosage du glucose et du lactate synovial dans l'arthrite aiguë. Les objectifs secondaires étaient d'évaluer l'intérêt pratique et les performances diagnostiques des bandelettes urinaires et du glucomètre dans le liquide synovial.

\section{Méthodes}

\section{2-1- Conception et population de l'étude.}

Une étude transversale monocentrique a été menée du 21/01/2015 au 30/06/2018 dans le Service de Rhumatologie du CHU de Rennes, France. Les liquides synoviaux ont été recueillis de manière prospective dans notre service de Rhumatologie (cohorte SYNOLACTATE). Les critères d'inclusion étaient : âge > 18 ans, épanchement articulaire sur articulation native, non traumatique évoluant depuis moins de 30 jours. Cette étude a reçu l'approbation du comité d'éthique de notre établissement. (Notification :16.96)

\section{2-2 Protocole de l'étude et mesures}

Les liquides synoviaux ont été obtenus par ponction articulaire. Les tests suivants ont été réalisés : analyse bactériologique des liquides synoviaux avec coloration de Gram, recherche de microcristaux, globules blancs synoviaux et dosages du lactate et du glucose. À compter du 20/05/2016, une analyse par deux bandelettes urinaires Combur10® UX strips et l'analyseur d'urine Urisys $1100 \circledast$ (F. Hoffmann-La Roche AG, Basel, Suisse) a été menée dans le liquide synovial. Puis, à partir du 28/12/2017, une mesure de la glycosynovie avec un glucomètre (Accu-Chek® performa) a été effectuée dans le liquide synovial. Le dosage du glucose et du lactate a été réalisé selon la technique du laboratoire de biochimie.

\section{2-3 Diagnostics et définitions de cas dans l'étude.}


L'arthrite septique a été définie selon les critères de Newman (2) et l'analyse du liquide synovial lorsqu'un des trois résultats suivants a été retrouvé : 1) mise en évidence d'un germe dans le liquide synovial ;2) mise en évidence d'un germe dans les hémocultures avec clinique typique d'arthrite septique ; 3) arthrocentèse révélant un liquide synovial purulent avec clinique typique d'arthrite septique, absence de cristaux et absence d'autre diagnostic. Dans certains cas, nous avons envisagé la possibilité d'une contamination bactérienne lorsque la situation ne correspondait pas à une étiologie septique et que la bactérie n'était pas pathogène (i.e. Cutibacterium acnes, Coagulase-negative Staphylococcus, Corynebacterium sp.). Dans ces cas-là, nous avons considéré les liquides synoviaux comme non septiques lorsque l'évolution clinique, biologique et radiographique était favorable sans recours à une antibiothérapie.

D'autres diagnostics ont été envisagés basés sur la présence ou l'absence de cristaux à la microscopie optique à lumière polarisée. En l'absence de microcristaux, une évaluation immunologique a été réalisée (anticorps antipeptides cycliques citrullinés (CCP), facteur rhumatoïde (FR) et anticorps anti-nucléaires (AAN)). Les critères ACR/EULAR 2010 (29) ont été utilisés pour classer les patients atteints de polyarthrite rhumatoïde (PR). Les critères ASAS (30) ont permis de classer les patients atteints de spondyloarthrite $(\mathrm{SpA})$ axiale et/ou périphérique. L'arthrite réactionnelle a été diagnostiquée comme étant une arthrite non-septique survenant au cours du mois qui suivait l'infection urétrale ou entéroinvasive (Chlamydia sp., Yersinia sp., Campylobacter sp....). L'arthrite microcristalline a été définie par la présence de cristaux d'urates de sodium (arthrite goutteuse) ou de cristaux de pyrophosphate de calcium (arthrite à dépôts de PCC), une culture bactériologique négative et une évolution favorable sous traitement adapté.

Le diagnostic final a été évalué par le médecin en charge du patient.

\subsection{Analyse statistique.}

L'analyse statistique a été réalisée au moyen du logiciel SPSS 20.0. Une valeur $p<0.05$ a été considérée comme statistiquement significative.

Les variables quantitatives continues ont été exprimées en moyenne \pm écarts-type ou médiane \pm écart interquartile $(\mathrm{IQR})$ après vérification de leur distribution Gaussienne ou non. Les variables gaussiennes dans notre population de patients étaient : âge, dosage du glucose synovial par glucomètre et dose moyenne de corticoïdes. Les autres variables ne suivaient pas une distribution gaussienne selon le test de Kolmogorov-Smirnov. La comparaison de ces variables ( $k$ étant le nombre de groupes de variables) a été effectuée par le test $t$ de Student (pour les distributions gaussiennes), le test non paramétrique $U$ de Mann-Whitney $(k=2)$ ou le test de Kruskal-Wallis $(k>2)$ (pour les distributions non gaussiennes). Les variables qualitatives ont été exprimées en nombre absolu et en pourcentage et leur comparaison réalisée avec un test de chi-2 ou le test exact de Fisher si l'un des nombres théoriques dans le tableau des contingences était inférieur à 5 . Pour les variables qualitatives $k>2$, un ajustement de Bonferroni a été effectué. Toutes les données manquantes ont été considérées comme négatives.

Pour évaluer les performances diagnostiques, les courbes ROC (Receiver Operating Characteristic) avec l'aire sous la courbe (AUC), les sensibilités (Se), les spécificités (Sp), les rapports de vraisemblance positifs $(\mathrm{RV}+)$ et négatifs (RV-) ainsi que leurs intervalles de confiance à $95 \%$ ont été calculés. Les tableaux de contingence ont également été produits avec évaluation de l'accord. La corrélation entre deux variables quantitatives a été calculée au moyen du coefficient de corrélation rho de Spearman.

\section{Résultats}

\subsection{Caractéristiques de la population étudiée}

Parmi les 251 liquides synoviaux analysés, 18 ont été exclus : 12 avec GBsyn <2000/mm3 (arthrose), 4 avec données manquantes, 1 n'était pas une arthrite sur articulation native, 1 liquide inclus 2 fois pour la même articulation (Figure 1). 
Ont été inclus dans l'étude 233 liquides synoviaux provenant de 226 patients. Pour 7 patients nous avions 2 liquides synoviaux différents. L'âge moyen de ces patients s'élevait à $61.8 \pm 20.2$, et $151(64.8 \%)$ étaient des hommes. Les ponctions articulaires ont été réalisées majoritairement sur le genou $(71.7 \%)$. Selon les critères définis cidessus, 25 arthrites ont été considérées comme septiques et 208 comme non bactériennes.

On notait un nombre significativement supérieur de cirrhoses dans le groupe des arthrites septiques par rapport au groupe des arthrites non septiques $(p<0.001)$ mais les autres comorbidités cliniques étaient comparables entre les deux groupes (Tableau 1). Dans $18.5 \%$ des cas, une antibiothérapie avait été débutée avant la ponction articulaire.

Staphylococcus sp. était l'organisme le plus fréquemment isolé, dans 11 cas. Streptococcus sp. a été retrouvé dans 6 cas, les bacilles à Gram négatifs dans 5 et le gonocoque dans 1 cas. Dans 2 cas, aucune bactérie n'a été mise en évidence mais le médecin a approuvé le diagnostic positif.

Parmi les 208 échantillons d'arthrite non bactérienne, 104 (50\%) étaient des arthrites microcristallines. Ont été relevés $15 \mathrm{PR}, 8 \mathrm{SpA}, 6$ arthrites réactionnelles, 57 arthrites aiguës d'origine indéterminée et 18 arthrites de causes plus rares.

\section{2- Performances du glucose et du lactate}

Les taux médians du lactate synovial et du ratio lactate/glucose étaient significativement plus hauts et la médiane des taux de glucose synovial était plus basse parmi les arthrites septiques que dans les arthrites aiguës non septiques (Tableau 1/Figure 2). Lorsque l'on examine l'AUC, la performance diagnostique était plus élevée pour le ratio lactate/glucose (0.859 [0.77-0.94]) que pour les taux de lactate ou de glucose calculés séparément (Figure 3).

La valeur seuil idéale du ratio lactate/glucose permettant de différencier l'arthrite septique de l'arthrite non septique était de 5 Se 52 \% [0.34-0.7], Sp 98.1\% [0.95-0.99], RV+ 27.0 [9.50-76.0], RV- [0.33-0.74]). (Tableau 2)

Les performances des autres variables sont détaillées dans la documentation [Annexe A, Tableau S1; voir le matériel complémentaire accompagnant la version en ligne de cet article]. En créant des tableaux de contingence pour le lactate, le glucose et le ratio lactate/glucose, les valeurs seuil pour

l'utilisation de ces paramètres ont été définies. (Annexe A, Tableau S2).

\subsection{Faux négatifs}

Parmi les 25 arthrites septiques (Tableau 3), 16 n'ont pas été discriminées par les paramètres glucose et lactate définissant ainsi des faux négatifs. Parmi ces 16 liquides synoviaux, nous avons observé 5 bacilles à gram négatif (BGN), 1 Neisseria gonorrhoeae, 8 cocci à gram positif comprenant 6 Staphylococcus aureus, 2 Streptococcus sp. et 2 arthrites septiques non documentées.

Les 5 arthrites septiques à Gram négatif affichaient un taux de lactate élevé (entre 8.77 et $17.68 \mathrm{mmol} / \mathrm{L}$ ) avec un taux de glucose non abaissé (non inférieur à $2 \mathrm{mmol} / \mathrm{L}$ ). II s'agissait de 5 arthrites septiques à BGN sans diminution du taux de glucose synovial ( 0 BGN vs 11 Gram positif (64.7 \%) pour un seuil de glucose < $1.8 \mathrm{mmol} / \mathrm{L}, \mathrm{p}=0.035$ ). (Annexe A, Figure S1) Parmi les faux négatifs, des particularités ont été observées. Pour trois arthrites septiques, le volume du liquide synovial aspiré était inférieur à $1 \mathrm{ml}$. Dans deux cas d'arthrite septique, l'antibiothérapie a été débutée avant la ponction. Dans un cas, la culture s'est avérée positive mais avec un inoculum bactérien de petite taille ( $<10$ colonies/boîte)

Parmi les 2 cas d'arthrites septiques non documentées, Streptococcus pneumoniae avec antigénurie positive a été fortement suspecté dans un cas. Dans le second cas, le caractère septique de l'arthrite a été fortement suspecté chez un patient immunodéprimé sous chimiothérapie. L'évolution, initialement défavorable sous ceftriaxone, a montré une amélioration après traitement par piperacilline-tazobactam et vancomycine.

\subsection{Faux positifs}


En utilisant le ratio lactate/glucose $>5$ dérivé de notre tableau, 4 cas de faux positifs ont été retrouvés (1 PR, 2 arthrites à CPP et 1 goutte). Pour un seuil de lactate synovial $\geq 10 \mathrm{mmol} / \mathrm{L}$, nous avons compté 2 cas de faux positifs (arthrite à CPP pour les 2 cas). Pour un seuil de glucose synovial $<1.8 \mathrm{mmol} / \mathrm{L}$, nous avons observé 10 cas de liquides synoviaux non septiques : 3 arthrites à dépôts de CPP, 1 goutte, 1 PR (avec très faible taux de glucose $0.1 \mathrm{mmol} / \mathrm{L}), 2$ arthrites réactionnelles et 3 cas d'arthrites indéterminées. Aucun cas de faux positif n'a été observé pour un seuil de lactate synovial de $11.5 \mathrm{mmol} / \mathrm{L}$.

\subsection{Corrélation entre les mesures par glucomètre, analyseur automatique et bandelettes urinaires}

Une très forte corrélation a été retrouvée entre le glucose synovial mesuré par glucomètre ou analyseur automatique $(n=30)$ avec le test rho de Spearman $\rho=0.93$. La corrélation entre le glucose synovial mesuré par un analyseur automatique et les bandelettes urinaires $(n=128)$ était modérée selon le test rho de Spearman ()$=0.58$ (Annexe A, Figure S2). Le coefficient rho de Spearman pour la corrélation entre les bandelettes $n^{\circ} 1$ et $n^{\circ} 2$ était $\rho$ $=0.70$.

\section{Discussion}

Cette étude analysait les performances diagnostiques du dosage du lactate et du glucose dans le liquide synovial pour différencier les arthrites septiques des arthrites non septiques. Nous avons démontré les bonnes performances du ratio lactate/glucose synovial pour discriminer les arthrites septiques des autres formes d'arthrite $(A \cup C>0.85 \cdot R V+27.0)$.

A notre connaissance, il s'agit de la première étude décrivant les performances du ratio lactate/glucose synovial. Dans notre étude, les performances du ratio lactate/glucose étaient supérieures à celles de chaque composant étudié séparément. Pour simplifier la comparaison avec la littérature, nous proposons de comparer de manière successive nos résultats sur le lactate synovial puis sur le glucose.

\subsection{Performances du lactate}

Depuis 1970, de nombreuses études ont analysé les performances diagnostiques du dosage du lactate. Conformément à nos résultats, la spécificité du lactate synovial dans la littérature était excellente variant de $95 \%$ (seuil de $12 \mathrm{mmol} / \mathrm{L}$ ) (31) à $100 \%$ (seuil $\geq 10 \mathrm{mmol} / \mathrm{L}$ ) (32) pour le diagnostic d'arthrite septique. D'autre part, la sensibilité a montré une variation plus importante allant de $56 \%$ (seuil $\geq 10 \mathrm{mmol} / \mathrm{L}$ ) (24) à $100 \%$ (seuil de 12 $\mathrm{mmol} / \mathrm{L})(31)$.

Ces variations étaient probablement attribuables aux différences de population de ces études et aux nombreux cas d'arthrose qu'elles incluaient (31-33). La faible sensibilité retrouvée dans notre étude (44\% pour un seuil $\geq$ $10 \mathrm{mmol} / \mathrm{L}$ ) est liée aux caractéristiques de notre cohorte d'arthrites très aiguës (évolution moyenne de 5 jours). Cette cohorte excluait les liquides synoviaux ayant un nombre de GBsyn $<2000 / \mathrm{mm} 3$ (arthrose) et incluait dans le groupe témoin de nombreuses arthrites microcristallines très inflammatoires. En effet pour un seuil de lactate synovial $\geq 10 \mathrm{mmol} / \mathrm{L}$, nous avons observé 2 faux positifs pour des arthropathies à CPP, probablement liés au caractère très inflammatoire des arthrites microcristallines (34). Certains auteurs ont attribué ces taux élevés de lactate à une hypoxie du tissu synovial $(35,36)$. Dans notre étude, les taux de CRP ont été corrélés aux taux de lactate (Annexe A, Figure S3). En effet, plus intense est la réaction inflammatoire dans l'arthrite (CRP augmentée), plus élevé sera le degré d'hypoxie tissulaire (taux élevé de lactate synovial). Néanmoins, il convient de noter qu'aucune des arthrites non septiques de notre étude affichait un taux de lactate supérieur à $11.5 \mathrm{mmol} / \mathrm{L}$, proche du seuil de $12 \mathrm{mmol} / \mathrm{L}$ déjà rapporté dans la littérature (31).

Lenski et al.(14) ont défini un taux de lactate $<4.3 \mathrm{mmol} / \mathrm{L}$ pour exclure une arthrite septique. Nous n'avons retrouvé aucune valeur de lactate permettant de définir une arthrite comme étant non septique avec une pertinence nonclinique du rapport de vraissemblance négatif. Toutefois, les liquides synoviaux mécaniques (GBsyn $<2000 / \mathrm{mm} 3$ ) ont été exclus de notre étude afin de n'y inclure que les liquides synoviaux inflammatoires. 
Pour finir, nos données étaient proches des AUC rapportées dans des études récentes concernant les performances du lactate (AUC 0.86 (14) et AUC 0.90(23)). Dans notre étude, les performances du lactate ont été altérées par certaines bactéries, notamment Neisseria sp. qui n'a pas causé une augmentation des taux de lactate. Cette caractéristique bien connue de l'arthrite à Neisseria gonorrhoeae, est certainement liée au mécanisme d'aérobie stricte $(35,37)$. Certains de ces faux positifs pourraient également être expliqués par des difficultés techniques. Pour la réalisation des dosages, il était nécessaire d'obtenir au moins $1 \mathrm{ml}$ de liquide synovial. Cependant, dans certaines articulations comme la cheville ou le poignet, il n'a pas toujours été possible de ponctionner une telle quantité de liquide. L'utilisation du ratio lactate/glucose a permis de compenser les difficultés techniques liées au faible volume et aux dilutions moins précises.

\subsection{Performances du glucose}

De précédentes études ont rapporté les performances du glucose synovial pour le diagnostic d'arthrite septique (AUC 0.70(14), 0.85(23), et AUC 0.96(26)).

Omar et al. (26) ont retrouvé un seuil de glucose synovial $<1.4 \mathrm{mmol} / \mathrm{L}$ pour discriminer l'arthrite septique avec une sensibilité de $100 \%$ et une spécificité de $92 \%$. Nous n'avons pas retrouvé une telle performance dans notre population. Là encore, la population de cette étude était très différente de la nôtre, avec $41 \%$ d'arthroses dans le groupe témoin. Comme mentionné précédemment, l'hypoxie tissulaire pourrait expliquer l'augmentation du lactate et le métabolisme anaérobie des synoviocytes pourrait être à l'origine de la consommation de glucose.

Par ailleurs, Omar et al. (26) ont exclu les patients ayant reçu une antibiothérapie avant la réalisation de la ponction articulaire alors que ces patients représentaient $20 \%$ de notre population. L'hypothèse selon laquelle l'antibiothérapie administrée avant la ponction pourrait réduire la taille de l'inoculum bactérien et, par là même, les taux de glucose nous semble intéressante. Toutefois, nous n'avons pas été en mesure de démontrer ce résultat en raison de la faible proportion d'arthrites septiques dans notre échantillon. Dans notre étude, les bactéries à Gram négatif ont été associées à une augmentation du lactate synovial sans baisse du glucose. Nous n'avons pas retrouvé de tels résultats dans la littérature (ex. dans l'étude d'Omar et al (26) l'absence de bacilles à Gram négatif ne permettait pas d'établir une comparaison). Une des explications possibles réside dans la différence de la voie métabolique des hydrates de carbone de la bactérie.

Ainsi, nous avons démontré, conformément au rapport de vraisemblance positif, que le seuil le plus discriminant pour l'arthrite septique dans une population d'arthrites aiguës était $<1.8 \mathrm{mmol} / \mathrm{L}$.

Les objectifs secondaires étaient d'évaluer l'intérêt pratique et les performances diagnostiques des bandelettes urinaires et du glucomètre dans le liquide synovial. Une forte corrélation a été retrouvée entre les taux de glucose mesurés par glucomètre et ceux mesurés par analyseur automatique (rho de Spearman $\rho=0.93$ ). Ce résultat confirmait l'excellente corrélation pour le dosage rapide du glucose synovial dans de précédentes études $(26,28)$. En ce qui concerne les bandelettes urinaires, les résultats obtenus étaient moins nets. Ces données encouragent l'abandon des bandelettes urinaires à la faveur de la plus grande précision et reproductibilité du glucomètre. Cependant, l'utilisation du glucomètre pourrait être impactée par les difficultés techniques liées par exemple à l'hyperviscosité du liquide synovial (27).

Le premier point fort de notre étude résidait dans la méthodologie utilisée, à savoir le recueil prospectif de liquides articulaires incluant des patients ayant reçu une antibiothérapie avant la ponction. II s'agissait de la plus grosse cohorte dans la littérature de liquides synoviaux d'articulations natives recueillis de manière prospective. Tous les patients ont été inclus dans cette étude pragmatique en vie réelle malgré la présence de données manquantes. Dans ce contexte, la coloration de Gram a été positive dans $16 \%$ des cas seulement, proportion plus faible que celle retrouvée dans la littérature (11). L'arthrite a fait l'objet d'une prise en charge rapide avec une ponction réalisée dans un délai inférieur à 5 jours ce qui explique probablement la petite taille de l'inoculum bactérien. Ce résultat pourrait également être attribué à l'antibiothérapie administrée avant la ponction dans $20 \%$ des cas. Du fait de la petite taille de notre échantillon, il ne nous a pas été possible de détecter une éventuelle différence entre les taux de lactate et de glucose avec ou sans antibiothérapie préalable. 
Plusieurs limites peuvent être mentionnées. 1) La proportion d'arthrites septiques était faible $(10.7 \%)$ comparativement aux $15 \%$ habituellement rapportés (1). Nous avons fait le choix d'inclure toutes les arthrites sur articulations natives évoluant depuis moins de 30 jours, sans critères d'exclusion, mais exigeant un volume de liquide synovial minimum pour l'analyse complète. Les arthrites septiques localisées aux petites articulations ayant un faible volume de liquide synovial n'ont pas été incluses ce qui explique certainement la prévalence observée dans notre étude. 2) Nous avons rapporté plusieurs cas de taux de glucose très élevés que les $10 \%$ de patients diabétiques ne peuvent à eux seuls expliquer. Nous avons utilisé le même processus d'analyse que celui décrit dans d'autres études (26) sans toutefois établir de comparaison entre les taux de glucose synovial et la glycémie capillaire. 3) La technique utilisée nous a permis de doser uniquement le L-Lactate $(25,38,39)$. Or, certaines bactéries produisent du D-Lactate plutôt que du L-Lactate ce qui pourrait en partie expliquer les taux relativement bas de lactate. Néanmoins, cette explication doit être envisagée avec prudence car dans notre étude, les faux négatifs retrouvés avec le lactate étaient moins nombreux que ceux du glucose, en particulier les bacilles à Gram négatif.

En conclusion, les performances du dosage du glucose et du lactate, et plus particulièrement du ratio lactate/glucose étaient bonnes et permettaient de discriminer les arthrites septiques des autres arthrites aiguës.

\section{Annexe A. Matériel complémentaire}

Du matériel complémentaire (Tableau S1-S2, Fig. S1-S3) accompagnant cet article est disponible sur ...

Déclaration d'intérêts : les auteurs ne déclarent aucun conflit d'intérêts en lien avec ce travail. Cette recherche n'a bénéficié d'aucun financement de la part d'une organisation publique, commerciale ou à but non lucratif. 


\section{Références}

1. Gupta MN, Sturrock RD, Field M. A prospective 2-year study of 75 patients with adult-onset septic arthritis. Rheumatology. 2001;40(1):24-30.

2. Newman JH. Review of septic arthritis throughout the antibiotic era. Ann Rheum Dis. 1976;35(3):198205.

3. Kaandorp CJ, Krijnen P, Moens HJ, Habbema JD, van Schaardenburg D. The outcome of bacterial arthritis: a prospective community-based study. Arthritis Rheum. 1997;40(5):884-92.

4. Weston VC, Jones AC, Bradbury N, Fawthrop F, Doherty M. Clinical features and outcome of septic arthritis in a single UK Health District 1982-1991. Ann Rheum Dis. 1999;58(4):214-9.

5. Hassan AS, Rao A, Manadan AM, Block JA. Peripheral Bacterial Septic Arthritis: Review of Diagnosis and Management. JCR J Clin Rheumatol. 2017;23(8):435-42.

6. Mathews CJ, Weston VC, Jones A, Field M, Coakley G. Bacterial septic arthritis in adults. Lancet Lond Engl. 2010;375(9717):846-55.

7. Ward PCJ. Interpretation of synovial fluid data. Postgrad Med. 1980 Sep 1;68(3):175-84.

8. Pavic K, Pandya J, Sebak S, Shetty A, Spencer D, Manolios NN. Acute arthritis: predictive factors and current practice in the approach to diagnosis and management across two hospitals in Sydney. Intern Med J. 2018;48:1087-95.

9. Prior-Español Á, García-Mira Y, Mínguez S, Martínez-Morillo M, Gifre L, Mateo L. Coexistence of septic and crystal-induced arthritis: A diagnostic challenge. A report of 25 cases. Reumatol Clin. $2019 ; 15: e 81-5$

10. Balderia PG, Pomerantz S, Fischer R. Acute bacterial arthritis: how long should you wait for culture results? J Clin Rheumatol Pract Rep Rheum Musculoskelet Dis. 2015;21(4):196-8.

11. Faraj AA, Omonbude OD, Godwin P. Gram staining in the diagnosis of acute septic arthritis. Acta Orthop Belg. 2002;68(4):388-91.

12. Stirling $P$, Tahir $M$, Atkinson HD. The limitations of Gram-stain microscopy of synovial fluid in concomitant septic and crystal arthritis. Curr Rheumatol Rev. 2018;255-7.

13. McGillicuddy DC, Shah KH, Friedberg RP, Nathanson LA, Edlow JA. How sensitive is the synovial fluid white blood cell count in diagnosing septic arthritis? Am J Emerg Med. 2007;25(7):749-52.

14. Lenski M, Scherer MA. The significance of interleukin- 6 and lactate in the synovial fluid for diagnosing native septic arthritis. Acta Orthop Belg. 2014;80(1):18-25.

15. Ferreyra M, Coiffier G, Albert J-D, David C, Perdriger A, Guggenbuhl P. Combining cytology and microcrystal detection in nonpurulent joint fluid benefits the diagnosis of septic arthritis. Jt Bone Spine Rev Rhum. 2017;84(1):65-70.

16. Shmerling RH, Delbanco TL, Tosteson AN, Trentham DE. Synovial fluid tests. What should be ordered? JAMA. 1990;264(8):1009-14.

17. Carpenter CR, Schuur JD, Everett WW, Pines JM. Evidence-based Diagnostics: Adult Septic Arthritis. Acad Emerg Med Off J Soc Acad Emerg Med. 2011;18(8):781-96. 
18. Talebi-Taher M, Shirani F, Nikanjam N, Shekarabi M. Septic versus inflammatory arthritis: discriminating the ability of serum inflammatory markers. Rheumatol Int. $2013 ; 33(2): 319-24$.

19. Saeed K, Dryden M, Sitjar A, White G. Measuring synovial fluid procalcitonin levels in distinguishing cases of septic arthritis, including prosthetic joints, from other causes of arthritis and aseptic loosening. Infection. $2013 ; 41(4): 845-9$.

20. Wang C, Zhong DA, Liao Q, Kong L, Liu A, Xiao H. Procalcitonin levels in fresh serum and fresh synovial fluid for the differential diagnosis of knee septic arthritis from rheumatoid arthritis, osteoarthritis and gouty arthritis. Exp Ther Med. 2014;8(4):1075-80.

21. Kopterides P. Synovial lactic acid and septic arthritis. JAMA. 2007 Jul 4;298(1):40; author reply 40.

22. Reimer LG. Approach to the analysis of body fluids for the detection of infection. Clin Lab Med. 1985;5(2):209-222.

23. Lenski M, Scherer MA. Analysis of synovial inflammatory markers to differ infectious from gouty arthritis. Clin Biochem. 2014;47(1-2):49-55.

24. Brook I. The importance of lactic acid levels in body fluids in the detection of bacterial infections. Rev Infect Dis. 1981;3(3):470-8.

25. Marcos MA, Vila J, Gratacos J, Brancos MA, Jimenez de Anta MT. Determination of D-lactate concentration for rapid diagnosis of bacterial infections of body fluids. Eur J Clin Microbiol Infect Dis Off Publ Eur Soc Clin Microbiol. 1991;10(11):966-9.

26. Omar M, Reichling M, Liodakis E, Ettinger M, Guenther D, Decker S, et al. Rapid exclusion of bacterial arthritis using a glucometer. Clin Rheumatol. 2017;36(3):591-8.

27. Coiffier G, Pollet S, Albert J-D, Perdriger A, Guggenbuhl P, Chales G. Usefulness and limitations of rapid urine dipstick testing for joint-fluid analysis. Prospective single-center study of 98 specimens. Jt Bone Spine Rev Rhum. 2013;80(6):604-7.

28. Omar M, Ettinger M, Reichling M, Petri M, Lichtinghagen R, Guenther D, et al. Preliminary results of a new test for rapid diagnosis of septic arthritis with use of leukocyte esterase and glucose reagent strips. J Bone Joint Surg Am. 2014;96(24):2032-7.

29. Aletaha D, Neogi T, Silman AJ, Funovits J, Felson DT, Bingham CO, et al. 2010 Rheumatoid arthritis classification criteria: an American College of Rheumatology/European League Against Rheumatism collaborative initiative. Arthritis Rheum. 2010;62(9):2569-81.

30. Sieper J, Rudwaleit M, Baraliakos X, Brandt J, Braun J, Burgos-Vargas R, et al. The Assessment of SpondyloArthritis international Society (ASAS) handbook: a guide to assess spondyloarthritis. Ann Rheum Dis. 2009;68 Suppl 2:ii1-44.

31. Riordan T, Doyle D, Tabaqchali S. Synovial fluid lactic acid measurement in the diagnosis and management of septic arthritis. J Clin Pathol. 1982;35(4):390-4.

32. Mossman SS, Coleman JM, Gow PJ. Synovial fluid lactic acid in septic arthritis. N Z Med J. 1981;93(678):115-7.

33. Brook I, Reza MJ, Bricknell KS, Bluestone R, Finegold SM. Synovial fluid lactic acid. A diagnostic aid in septic arthritis. Arthritis Rheum. $1978 ; 21(7): 774-9$.

34. Gerster JC, Gobelet C. Synovial fluid lactic acid in acute and chronic pyrophosphate arthropathy and in osteoarthritis. Clin Rheumatol. 1988 ;7(2):197-9.

35. Riley TV. Synovial fluid lactic acid levels in septic arthritis. Pathology (Phila). $1981 ; 13(1): 69-71$. 
36. James MJ, Cleland LG, Rofe AM. Determinants of synovial fluid lactate concentration. J Rheumatol. $1992 ; 19(7): 1107-10$.

37. Kirwan JR. Synovial fluid lactate in sep1. Gupta MN, Sturrock RD, Field M. A prospective 2-year study of 75 patients with adult-onset septic arthritis. Rheumatology. $2001 ; 40(1): 24-30$.

38. Kortekangas P, Peltola O, Toivanen A, Aro HT. Synovial fluid L-lactic acid in acute arthritis of the adult knee joint. Scand J Rheumatol. 1995;24(2):98-101.

39. Smith SM, Eng RH, Campos JM, Chmel H. D-lactic acid measurements in the diagnosis of bacterial infections. J Clin Microbiol. 1989 ;27(3):385-8.

tic arthritis. Lancet Lond Engl. 1982 Feb 20;1(8269):457.

38. Kortekangas P, Peltola O, Toivanen A, Aro HT. Synovial fluid L-lactic acid in acute arthritis of the adult knee joint. Scand J Rheumatol. 1995;24(2):98-101.

39. Smith SM, Eng RH, Campos JM, Chmel H. D-lactic acid measurements in the diagnosis of bacterial infections. J Clin Microbiol. 1989 ;27(3):385-8. 
Figure 1. Diagramme de flux des liquides synoviaux inclus dans cette étude

Figure 2. Courbes ROC

Courbes ROC et AUC correspondantes des marqueurs permettant de différencier les arthrites septiques des autres arthrites aiguës. Une AUC proche de 1.0 indique une sensibilité et une spécificité élevées.

Lactate synovial (AUC : 0.795). Ratio lactate/glucose synovial : (AUC : 0.859). Glucose synovial (AUC : 0.833)

Figure 3. Diagramme de dispersion. Taux de lactate (A), glucose (B) et ratio lactate/glucose (C) dans les arthrites septiques, arthrites microcristallines et arthrites "Non-non".

Diagramme de dispersion. Comparaison des taux de lactate synovial, glucose synovial et ratio lactate/glucose entre les arthrites septiques, arthrites microcristallines et arthrites non septiques et non microcristallines (« non-non »). La ligne horizontale en pointillés indique le seuil choisi pour chaque paramètre. 


\section{1 liquides synoviaux analysés}

12 arthroses

2 liquides sans aucune donnée

2 analyses du glucose et du lactate non valides

1 infection sur prothèse

1 liquide inclus 2 fois dans la même journée et pour la même articulation

233 liquides synoviaux inclus 


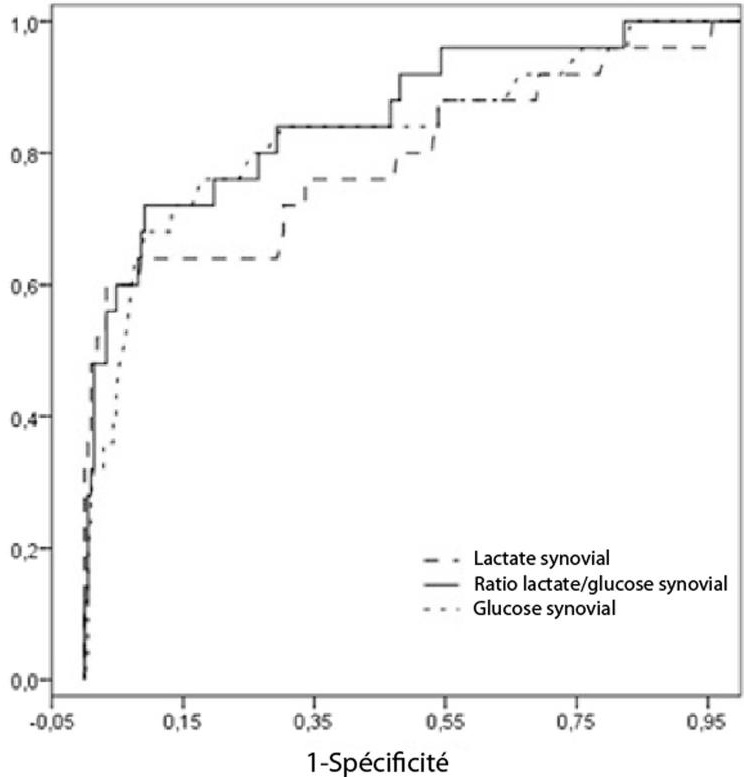


Tableau 1. Caractéristiques des 233 arthrites aiguës avec analyse du liquide synovial incluses dans l'étude avec comparaison entre les arthrites septiques $(n=25)$ et les arthrites non septiques $(n=208)$.

\begin{tabular}{|c|c|c|c|}
\hline & $\begin{array}{l}\text { Population globale } \\
n=233\end{array}$ & $\begin{array}{c}\text { Arthrite septique } \\
\quad n=25\end{array}$ & $\begin{array}{l}\text { Arthrite non septique } \\
\qquad n=208\end{array}$ \\
\hline Age (années \pm ET) & $61.8 \pm 20.2$ & $64.0 \pm 20.2$ & $61.6 \pm 20.2$ \\
\hline Sexe masculin & $151(64.8)$ & $20(80.0)$ & 131(63.0) \\
\hline \multicolumn{4}{|l|}{ Comorbidités } \\
\hline Rhumatisme inflammatoire & $80(34.3)$ & $5(20.0)$ & $75(36.1)$ \\
\hline Immunosuppression & $67(28.8)$ & $8(32.0)$ & $59(28.4)$ \\
\hline Cirrhose hépatique & $5(2.1)$ & $4(16.0)$ & $1(0.5)^{\prime}$ \\
\hline Diabète & $24(10.3)$ & $2(8.0)$ & $22(10.6$ \\
\hline Transplantation d'organe & $5(2.1)$ & $1(4.0)$ & $4(1.9)$ \\
\hline Cancer évolutif & $9(3.9)$ & $2(8.0)$ & $7(3.4)$ \\
\hline VIH & $1(0.4)$ & 0 & $1(0.5$ \\
\hline DMARDs & $24(10.3)$ & $2(8.0)$ & $22(10.6)$ \\
\hline Agents biologiques & $5(2.1)$ & $1(4.0)$ & $4(1.9)$ \\
\hline Chimiothérapie & $6(2.6)$ & $1(4.0)$ & $5(2.4)$ \\
\hline Glucocorticoïdes & $32(13.7)$ & 0 & $32(15.4)^{*}$ \\
\hline dose moyenne $(\mathrm{mg} / \mathrm{j} \pm \mathrm{ET})$ & $14.7 \pm 3.9$ & & \\
\hline \multicolumn{4}{|l|}{ Signes cliniques } \\
\hline Durée des symptômes & $5.0[3.0-10.0]$ & $6.0[2.0-14.0]$ & $5.0[3.0-10.0$ \\
\hline \multicolumn{4}{|l|}{ Nombre d'articulations } \\
\hline Mono & $159(68.2)$ & $19(76.0)$ & $140(67.3)$ \\
\hline Oligo & $51(24.5)$ & $6(24.0)$ & $45(21.6)$ \\
\hline Poly & $23(9.3)$ & 0 & $23(11.1)$ \\
\hline \multicolumn{4}{|l|}{ Articulations concernées } \\
\hline Genou & $167(71.7)$ & $14(56.0)$ & $153(73.6$ \\
\hline Cheville & $13(5.6)$ & $2(8.0)$ & $11(5.3)$ \\
\hline Hanche & $17(7.3)$ & $3(12.0)$ & $14(6.7)$ \\
\hline Épaule & $16(6.8)$ & $3(12.0)$ & $13(6.3)$ \\
\hline Coude & $16(6.8)$ & 0 & $16(7.7)$ \\
\hline Poignet & $5(1.7)$ & $3(12.0)$ & $1(0.5)^{*}$ \\
\hline Fièvre & $87(37.3)$ & $17(68.0)$ & $70(33.7)^{* * *}$ \\
\hline Antibiothérapie (<10 jours) & $43(18.5)$ & $9(36.0)$ & $34(16.3)^{* *}$ \\
\hline AINS $(<15$ jours $)$ & $25(10.7)$ & $4(16.0)$ & $21(10.1)$ \\
\hline \multicolumn{4}{|l|}{ Bilan sanguin } \\
\hline CRP & 82.8 [35.3-153] & $188.3[88.8-288]$ & 76.7 [29.8-132] \\
\hline Hémocultures positives ( $n=196)$ & $15(7.7)$ & $7(28.0)$ & $8(4.7)$ \\
\hline \multicolumn{4}{|l|}{ Liquide synovial } \\
\hline \multicolumn{4}{|l|}{ Macroscopie $(n=205)$} \\
\hline Purulent & $26(12.7)$ & $16(66.7)$ & $10(5.5)^{*}$ \\
\hline Trouble & $125(61.0)$ & $5(20.8)$ & $120(79.5)^{* *}$ \\
\hline Jaune et transparent & $37(18.0)$ & $2(8.3)$ & $35(19.3$ \\
\hline Inflammatoire & $17(8.3)$ & $1(4.2)$ & $16(8.8$ \\
\hline \multicolumn{4}{|l|}{ Microscopie } \\
\hline GB syn $\left(/ \mathrm{mm}^{3}\right)$ & $16500[6050-49000]$ & 79000 [15000-117000] & $13900[5400-43000]^{\prime}$ \\
\hline $\mathrm{EN}>50000 / \mathrm{mm}^{3}$ & $52(22.3)$ & $13(52.0)$ & $39(18.8)^{3}$ \\
\hline Polynucléaires neutrophiles (\%) & $87.0[72.5-92.0]$ & $90.5[83.8-94.0]$ & $86.0[67.0-92.0]^{* *}$ \\
\hline PNN $>90 \%$ & $81(34.7)$ & $13(52.0)$ & $68(29.2)^{*}$ \\
\hline Microcristaux & $108(46.3)$ & $4(16.0)$ & $104(50.0)^{* * *}$ \\
\hline \multicolumn{4}{|l|}{ Microbiologie } \\
\hline Coloration de Gram & $4(1.7)$ & $4(16.0)$ & 0 \\
\hline Culture bactériologique & $29(12.4)$ & $20(80.0)$ & $9(4.3)^{*}$ \\
\hline \multicolumn{4}{|l|}{ Biochimie } \\
\hline Glucose synovial (mmol/L) & $5.5[4.0-7.0]$ & $2.2[0.68-4.3]$ & $5.7[4.6-7.0]$ \\
\hline Lactate synovial (mmol/L) & $4.0[2.8-5.9]$ & 9.3 [4.4-16.3] & $3.7[2.7-5.5]$ \\
\hline Ratio lactate/glucose & $0.67[0.41-1.27]$ & $3.7[1.2-24.7]$ & $0.60[0.39-1.09]$ \\
\hline \multicolumn{4}{|l|}{ Bandelette urinaire $(n=129)$} \\
\hline Déficit en glucose & $22(17.1)$ & $10(76.9)$ & $12(10.3)^{\star *}$ \\
\hline Glycosurie & $97(75.2)$ & $3(23.1)$ & $94(81.0)^{* *}$ \\
\hline incertain & $10(7.8)$ & 0 & $10(8.6)$ \\
\hline
\end{tabular}

${ }^{*} p<0,001 ;{ }^{* *} p<0,05 ;{ }^{* * *} p<0,01$ 
Tableau 2. Performances du lactate, du glucose et du ratio lactate/glucose dans le liquide synovial pour le diagnostic d'arthrite septique.

\begin{tabular}{|c|c|c|c|c|c|c|}
\hline & AUC (IC 95\%) & Seuil & Se (IC 95\%) & Sp (IC 95\%) & $R V+(I C 95 \%)$ & RV- (IC $95 \%)$ \\
\hline Glucose (mmol/L) & $0.833(0.735-0.931)$ & $\begin{array}{l}\leq 1.0 \\
\leq 1.4 \\
\leq 1.8\end{array}$ & $\begin{array}{l}32 \%(0.17-0.52) \\
36 \%(0.20-0.55) \\
44 \%(0.27-0.63)\end{array}$ & $\begin{array}{l}99.0 \%(0.97-1.0) \\
97.1 \%(0.94-0.99) \\
95.2 \%(0.91-0.97)\end{array}$ & $\begin{array}{l}33.3(7.5-148) \\
12.5(4.8-32) \\
15.6(5.6-43)\end{array}$ & $\begin{array}{l}0.69(0.53-0.90) \\
0.66(0.49-0.89) \\
0.59(0.42-0.83)\end{array}$ \\
\hline Lactate $(\mathrm{mmol} / \mathrm{L})$ & $0.795(0.681-0.910)$ & $\begin{array}{l}\geq 11.0 \\
\geq 10.0 \\
\geq 9.5 \\
\geq 8.5 \\
<4.3\end{array}$ & $\begin{array}{l}36.0 \%(0.20- \\
0.55) \\
40.0 \%(0.23- \\
0.59) \\
48 \%(0.30-0.67) \\
60 \%(0.41-0.77) \\
76 \%(0.57-0.89)\end{array}$ & $\begin{array}{l}99.5 \%(0.97-1.00) \\
99.0 \%(0.97-1.00) \\
98.1 \%(0.95-0.99) \\
96.6 \%(0.93-0.98) \\
59.6 \%(0.53-0.66)\end{array}$ & $\begin{array}{l}74.9(9.9-566) \\
41.6(9.7-179) \\
25.0(8.7-71) \\
17.8(9.9-63) \\
1.9(1.4-2.5)\end{array}$ & $\begin{array}{l}0.64(0.48-0.86) \\
0.61(0.44-0.84) \\
0.53(0.36-0.77) \\
0.41(0.25-0.66) \\
0.40(0.20-0.82)\end{array}$ \\
\hline Ratio L/Glc & $0.859(0.772-0.945)$ & $\begin{array}{l}\geq 5.0 \\
<1\end{array}$ & $\begin{array}{l}52 \%(0.34-0.70) \\
80 \%(0.61-0.91)\end{array}$ & $\begin{array}{l}98.1 \%(0.95-0.99) \\
71.6 \%(0.65-0.77) \\
\end{array}$ & $\begin{array}{l}27.0(9.5-76) \\
2.8(2.1-3.8)\end{array}$ & $\begin{array}{l}0.49(0.33-0.74) \\
0.28(0.13-0.61) \\
\end{array}$ \\
\hline
\end{tabular}

Les valeurs entre parenthèses sont les intervalles de confiance à $95 \%$ correspondants. AUC: aire sous la courbe

Se : sensibilité, Sp : spécificité, $R V+$ : rapport de vraissemblance positif, $R V-$ : rapport de vraisemblance négatif 
Tableau 3. Charactéristiques des 25 arthrites septiques.

\begin{tabular}{|c|c|c|c|c|c|c|c|c|c|c|c|c|c|c|c|c|c|c|}
\hline & Sexe & Années & $\begin{array}{l}\text { Articulati } \\
\text { on }\end{array}$ & $\begin{array}{l}\text { Durée } \\
\text { (jours) }\end{array}$ & Fièvre & ATB & $\begin{array}{l}\text { CRP } \\
(\mathrm{mg} / \mathrm{l})\end{array}$ & $\begin{array}{l}\text { Nbre de } \\
\text { ponctions }\end{array}$ & Purulent & $\begin{array}{l}\text { GBsyn } \\
\left(/ \mathrm{mm}^{3}\right)\end{array}$ & $\begin{array}{l}\text { PMN } \\
(\%)\end{array}$ & Cristaux & GT & $\begin{array}{c}\text { Culture du } \\
\text { liquide synovial }\end{array}$ & Hémoculture & $\begin{array}{l}\text { Glucose } \\
(\mathrm{mmol} / \mathrm{L})\end{array}$ & $\begin{array}{l}\text { Lactate } \\
\text { (mmol/L) }\end{array}$ & $\begin{array}{c}\text { Ratio } \\
\text { Lact/Glc }\end{array}$ \\
\hline 1 & M & 46 & $\mathrm{P}$ & 2 & - & - & 157 & 1 & + & 200000 & 95 & - & - & N.gonorroeae & - & 2.20 & 5.25 & 2.4 \\
\hline 2 & $M$ & 58 & $\mathrm{H}$ & 15 & + & $+a$ & 364 & 1 & + & & 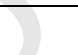 & - & - & MRSA & MRSA & 0.40 & 27.0 & 67.5 \\
\hline 3 & $\mathrm{~F}$ & 59 & G & 2 & + & - & 326 & 1 & + & 62500 & 94 & - & + & S.pneumoniae & - & 0.70 & 16.1 & 23.0 \\
\hline 4 & $M$ & 59 & $E$ & 8 & + & - & 83 & 1 & - & 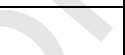 & 95 & - & - & MSSA & MSSA & 0.10 & 17.2 & 172 \\
\hline 5 & $M$ & 33 & C & 7 & + & $+a$ & 486 & 1 & + & +9 & & - & + & MSSA & - & 0.40 & 24.9 & 62.4 \\
\hline 6 & $M$ & 47 & $P$ & 7 & + & - & 26 & 1 & + & 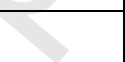 & & - & - & MSSA & - & 1.20 & 8.73 & 7.3 \\
\hline 7 & M & 86 & $C$ & 2 & + & - & 92 & 1 & - & 118000 & 84 & CPP & - & S.gordonii & S.gordonii & 2.50 & 5.20 & 2.1 \\
\hline 8 & M & 93 & $E$ & 10 & + & - & 103 & 2 & + & 350000 & 77 & - & - & MSSA & MSSA & 7.80 & 7.61 & 1.0 \\
\hline 9 & $M$ & 91 & G & 6 & + & $+c$ & 299 & 2 & - & 1200 & 89 & CPP & - & E.coli & - & 2.70 & 9.68 & 3.6 \\
\hline 10 & $M$ & 37 & G & 13 & + & $+d$ & 161 & 3 & - & 80000 & 96 & - & - & MSSA & - & 5.90 & 3.87 & 0.7 \\
\hline 11 & M & 18 & G & 1 & + & - & 99 & 2 & - & 16000 & 92 & - & - & MSSA & - & 7.00 & 2.50 & 0.4 \\
\hline 12 & $\mathrm{~F}$ & 89 & G & 7 & - & $+c$ & 195 & 2 & + & 80000 & 92 & CPP & - & - & E.Coli & 2.50 & 9.33 & 3.7 \\
\hline 13 & $\mathrm{M}$ & 79 & $E$ & 21 & - & $+\mathrm{b}, \mathrm{e}$ & 85 & 2 & + & 81000 & 100 & - & - & - & - & 4.60 & 2.93 & 0.6 \\
\hline 14 & $M$ & 79 & G & 15 & + & $+c$ & 189 & 2 & + & 7700 & 76 & - & - & - & S.dysgalactiae & 1.80 & 9.72 & 5.4 \\
\hline 15 & $M$ & 56 & $\mathrm{H}$ & 15 & + & - & 286 & 1 & + & & 88 & - & + & MSSA & MSSA & 0.50 & 14.8 & 29.8 \\
\hline 16 & $F$ & 86 & G & 2 & + & - & 355 & 2 & + & 116000 & 77 & - & + & S.dysgalactiae & - & 0.90 & 23.70 & 26.3 \\
\hline 17 & $M$ & 90 & G & 15 & -9 & $+c$ & 291 & 2 & + & & 92 & - & - & P.aeruginosa & - & 3.40 & 17.6 & 5.2 \\
\hline 18 & $\mathrm{M}$ & 58 & $G$ & 2 & - & - & 54 & 1 & - & 70000 & 91 & CPP & - & - & - & 4.80 & 10.1 & 2.1 \\
\hline 19 & $M$ & 82 & G & 4 & - & - & 262 & 1 & - & 78000 & 90 & - & - & S.dysgalactiae & - & 3.90 & 4.86 & 1.3 \\
\hline 20 & M & 52 & $\mathrm{H}$ & 3 & + & $+b$ & 243 & 3 & + & 450000 & 83 & - & - & MSSA & MSSA & 0.60 & 3.59 & 6.0 \\
\hline 21 & $\mathrm{~F}$ & 43 & $P$ & 3 & + & - & 6 & 1 & + & 100000 & 92 & - & - & $M S S A$ & - & 1.60 & 1.68 & 1.1 \\
\hline 22 & $M$ & 69 & G & 1 & + & - & 188 & 1 & + & & 84 & - & - & E.coli & - & 2.00 & 11,4 & 5.7 \\
\hline 23 & $\mathrm{~F}$ & 66 & G & 15 & - & - & 71 & 1 & - & 3800 & 82 & - & - & MSSA & - & 6.50 & 3,61 & 0.6 \\
\hline 24 & $M$ & 62 & G & 6 & - & - & 103 & 2 & - & 12000 & 90 & - & - & S.gordonii & - & 0.40 & 16,4 & 41.2 \\
\hline 25 & $M$ & 62 & G & 5 & + & - & 230 & 4 & + & 64000 & 94 & - & - & H.parainfluenza & & 2.90 & 8,77 & 3.0 \\
\hline
\end{tabular}

${ }^{a}$ Cloxacilline, ${ }^{b}$ Amoxiciline/Clavulanate, ${ }^{c}$ Ceftriaxone, ${ }^{d}$ Clindamicine, ${ }^{e}$ Fluoroquinolones ; P : Poignet ; H : Hanche ; G : Genou ; E : Epaule ; C : Cheville ; CPP : Cristaux de pyrophosphate de calcium

GBsyn = globules blancs synoviaux, GT = coloration de Gram, MSSA = Staphylococcus aureus sensible à la méticilline, MRSA = Staphylococcus aureus résistant à la méticilline 